\title{
Understanding 2D Crystal Vertical Heterostructures at the Atomic Scale Using Advanced Scanning Transmission Electron Microscopy
}

Sarah J. Haigh ${ }^{1,2}$, Aidan P. Rooney ${ }^{1,2}$, Tom J.A. Slater ${ }^{1}$, Eric Prestat ${ }^{1}$, Ekaterina Khestanova ${ }^{2}$, Rob Dryfe $^{3}$, Matej Velický ${ }^{3}$, Roman V. Gorbachev ${ }^{2}$, Rada Boya ${ }^{2}$, Yang Cao ${ }^{2}$, Irina Grigorieva ${ }^{2}$, Kostya Novoselov $^{2}$, Fred Withers ${ }^{2}$, Andre K. Geim ${ }^{2}$

1. School of Materials, University of Manchester, Manchester, M13 9PL, UK

2. National Graphene Institute, University of Manchester, Manchester, M13 9PL, UK

3. School of Chemistry, University of Manchester, Manchester, M13 9PL, UK.

The emerging area of two dimensional (2D) materials has attracted a great deal of scientific attention in recent years. Like graphene, these materials can be exfoliated to single atom thickness and can then be mechanically layered together to create new van der Waals crystals with bespoke properties. However the performance of such materials is strongly dependent on the quality of the crystals and the interfaces between different crystals. Cross sectional imaging using atomic resolution transmission electron microscopy (TEM) and scanning transmission electron microscopy (STEM) is the only technique able to characterize the nature of buried interfaces in these engineered van der Waals crystals [1].

We have been developing specimen preparation and image analysis methods for investigating the structure of 2D crystal heterostructures at the atomic scale [1,2]. I will present work demonstrating that cross sectional STEM imaging, coupled with energy dispersive X-ray (EDX) and electron energy loss (EEL) spectrum imaging, can be used to provide new insights to interpret the optical and electronic properties of such systems [1,2]. For example, we have studied light emitting diode (LED) devices (Figure 1), produced by mechanical exfoliation and subsequent stacking of up to 13 different 2D crystals, including $\mathrm{MoS}_{2}$ or $\mathrm{WS}_{2}$ monolayer quantum wells [3]. Using cross sectional STEM EDX and EEL spectrum imaging we reveal that the crystal interfaces of such devices are atomically flat and provide detailed structural information regarding the thickness of individual layers which is essential to understanding the photoluminescence and electroluminescence results obtained.

We have also applied this cross sectional imaging technique to $2 \mathrm{D}$ crystal heterostructures where individual planes of atoms have been effectively removed to produce nanochannels. I will demonstrate recent work where cross sectional imaging has aided the development of such capillary systems and the application of these nanochannels to help understand water flow at the nanoscale. Specifically characterizing the unexpectedly high fluid flow rates that are observed for few atomic layer graphene channel heights compared to larger channels [4]. We further use atomic resolution TEM imaging to reveal how confinement in such channels provides high pressure which can drive chemical transformations in aqueous salts [5].

Like our artificial 2D crystal heterostructures, some natural mineral phases are also composed of incommensurate 2D layers. I will present recent results showing the exfoliation of one such crystal, Franckeite, and the characterization of the excellent electrochemical properties for the exfoliated flakes, which offer greater promise for energy storage applications than traditional transition metal dichalcogenides (Figure 2) [6]. Finally I will discuss an exciting new area of 2D materials research which is developing around 2D crystals that are unstable in air. By harnessing the environmental protection provided by graphene encapsulation we can image air sensitive 2D materials like black 
phosphorus [7]. Using TEM combined with density functional theory we provide the first characterisation of atomic substitutional defects in the air sensitive monolayer superconductor $\mathrm{NbSe}_{2}$ as a first step to understanding the unexpected properties of this material $[8,9]$.

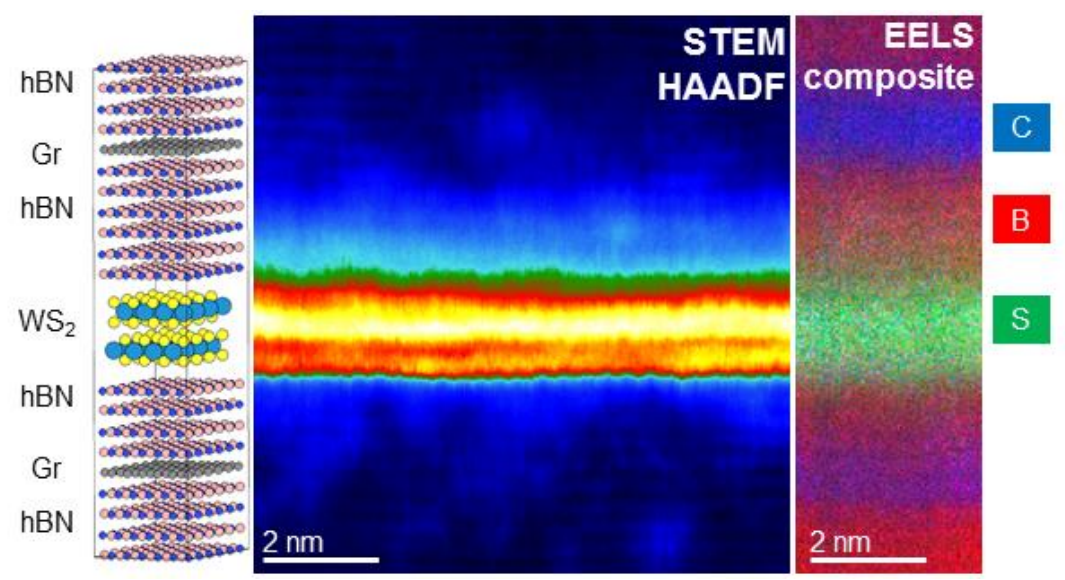

Figure 1. Cross sectional STEM imaging and EEL spectroscopy of a $\mathrm{WS}_{2}$ quantum well inside a boron nitride, graphene, boron nitride double sandwich. Similar to those described in ref [3].

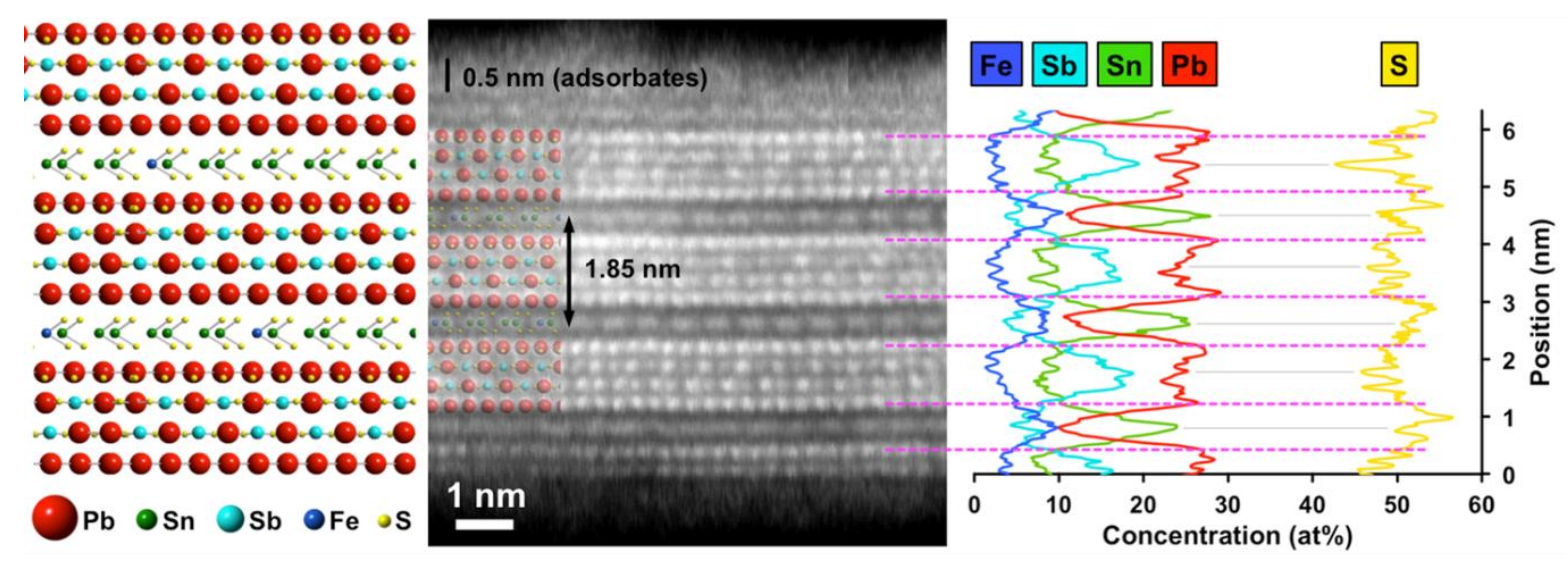

Figure 2. Cross sectional STEM imaging and EDX spectroscopy of a Franckeite crystal exfoliated to few layer thickness [6].

\section{References:}

[1] Haigh et al, Nature Materials 11, (2012) p. 764.

[2] Georgiou et al, Nature Nanotechnology 8, (2013) p. 100.

[3] Withers et al, Nature Materials, 14, (2015) p. 301

[4] Boya et al, Nature, 538, (2016) p. 222.

[5] Vasu et al, Nature Communications, 7, (2016), 12168.

[6] Velický et al, Nature Communications, 8, (2017), 14410.

[7] Cao et al, Nano Letters, 15, (2015) p. 4914

[8] Nguyen et al, ACS Nano, (2017) online article asap

[9] The authors acknowledge funding from the Engineering and Physical Sciences Research Council (EPSRC), Defence Threat Reduction Agency and European Research Council. 